\title{
Comparison of Negotiation Models for Cellular Network
}

\author{
Bapu Kabugade \\ Information Technology, \\ V. E. S. Institute of Technology, \\ Chembur, Mumbai
}

\author{
Mohit Shroff \\ Computer Engineering, \\ V. E. S. Institute of Technology \\ Chembur, Mumbai
}

\author{
Nupur Giri, Ph.D \\ Computer Engineering, \\ V. E. S. Institute of Technology \\ Chembur, Mumbai
}

\begin{abstract}
The work compares negotiation behaviors of Cellular Service Providers based on thrashing rate, message overhead and delay characteristics using four auction protocols viz. First Price Sealed Bid, Vickrey QoS, Vickrey utility and Dutch model.

It helps in establishing relationship between User Agent (UA) QoS and the choice of negotiation model in terms of thrashing rate and number of messages required to complete the negotiation process.
\end{abstract}

\section{General Terms}

Thrashing rate, Delay, Message overhead, Service Providers, Negotiation

\section{INTRODUCTION}

The liberalization of telecommunications networks is expected to lead to any-to-any scenario with any customer being free to buy services from any service provider, who in turn could buy network capacity from any network provider.

In designing the system for next generation networks, it is important to consider the business objectives of all parties involved (i.e. Customer, Service Provider and Network Provider). As pointed out in the Introduction, all parties will undoubtedly wish to maximize their return on investment. However, all parties will also be interested in making best use of scarce resources: the customers because they wish to maximize access to resources when they require them; the Network Providers and Service Providers because they need to strive to provide such availability to achieve customer satisfaction for their respective customers.

In systems composed of multiple autonomous agents, negotiation is a key form of interaction that enables groups of agents to arrive at a mutual agreement regarding some belief, goal or plan. Here, we have tried to explain how the behavior of agent changes when they switch between service providers at a faster rate i.e. thrashing. Other factors such as message overhead and delay are analyzed briefly.

\section{DESIGN AND FORMULAE}

\subsection{Thrashing}

Thrashing is defined as the migration of a user's subscription from one SPNA to another SPNA at a high rate. This causes unwanted delay in negotiation every time the user switches to a SPNA. The delay eventually increases to an extent that the call drops. In this paper, we have analyzed the amount of time for which a SPNA can retain a user's subscription under different traffic conditions and negotiation models viz. First Price sealed bid (FPSB), vickrey QoS model, vickrey utility model and the Dutch auction model.
In systems composed of multiple agents, negotiation is a key form of interaction among agents to arrive at a common conclusion which is fair and acceptable to all the agents participating in that negotiation process. This happens especially when agent behavior is not benevolent and they must convince others to act in certain ways.

Thrashing rate is defined as number of thrashing per unit time. In this paper, thrashing rate is calculated as the number of thrashings undergone by an auction model per minute.

Thrashing Rate $=\frac{\text { Number of negotiation }}{\frac{\sum \text { Simulation time }}{60}}$

Unit of measure is number of negotiations/minute.

Each model was simulated until it had undergone at least 10 negotiations. The total time taken to run the simulation was observed and applied in the formula above.

\subsection{Message Overhead (MO)}

Number of messages passed per negotiation is defined as message overhead. Calculation of message overhead for FPSB and Vickrey models as follows

When the current SPNA behaves as a coordinator SPNA [1], it sends (S-1) Call for Proposal (CFP) ACL message [3] for requesting their bids. The participating SPNAs in turn reply with their bids to the coordinator SPNA.

The coordinator SPNA selects the winning bidder based on the threshold formula of the respective auction model and informs the winning SPNA of the same. The winning SPNA then sends an acknowledgement to the coordinator SPNA. This concludes the auction process.

Calculation of message overhead for First Price Sealed Bid, Vickrey QoS and Vickrey Utility auction model is,

$$
\text { Message Overhead }=2 * S
$$

Where $\mathrm{S}$ is the number of service providers.

Calculation of message overhead for Dutch auction model is,

$$
\text { Message Overhead }=2\left[(s-1)+\sum_{i=1}^{n} m_{i}\right]
$$

Where $\mathrm{S}$ is the number of service providers, $\mathrm{n}$ is the number of negotiation rounds performed in the dutch model, $\mathrm{m}$ is the number of eligible service providers (SP) in that particular round. 
Figure 1 shows the ACL message [3,6] passed among the JADE [3] agents in the negotiation process.

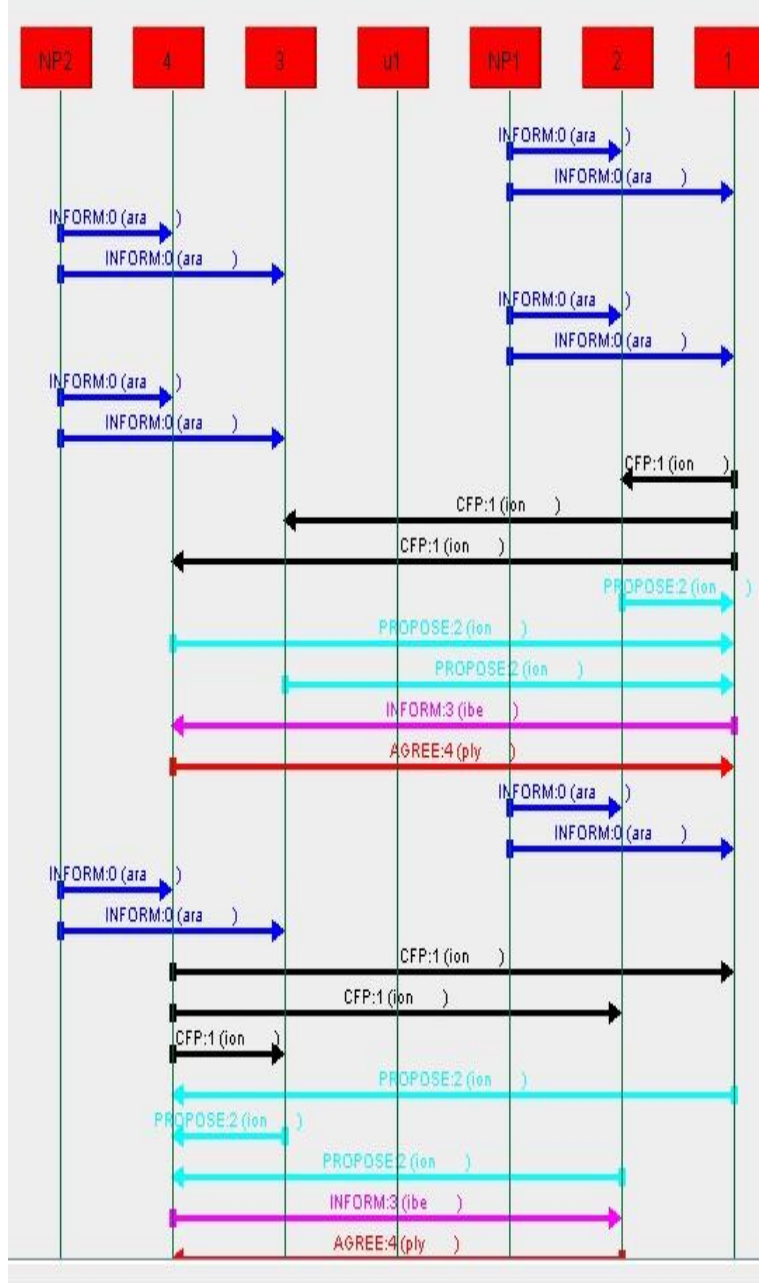

Fig 1. Interaction among JADE agents

Following are the ACL messages $[3,6]$ exchanged in the negotiation process by the various JADE agents:

\subsubsection{INFORM}

Every Network Provider Agent(NPA)[1] sends its network traffic values to all the Service Provider Agents (SPA) that are subscribed under that particular NPA using the INFORM message. These traffic values are used by the Service Provider Negotiation Agent (SPNA) to calculate the utility function and trigger an auction if the utility is below the threshold value desired by the user.

\subsection{2. $C F P$}

The Call For Proposal (CFP) message is sent by the current Service Provider Negotiation Agent who can no longer provide the desired service asked by the user i.e. its utility value is below the threshold. The current SPNA now acts as a coordinator Service Provider Negotiation Agent and monitors the auction process.

\subsubsection{PROPOSE}

The PROPOSE message is a reply from all the Service Provider Negotiation Agents which are participating in the auction process to the coordinator Service Provider Negotiation Agent. The reply message contains the bid value i.e. utility value for the user's subscription.

\subsubsection{INFORM}

This message is sent by the coordinator SPNA to the participating SPNA who has won the auction process.

\subsubsection{AGREE}

This message is an acknowledgement by the winning SPNA and is sent to the coordinator SPNA. Thus the auction process is completed and the user's subscription is transferred to the winning SPNA.

During the Dutch auction, the negotiation continues for multiple rounds until a single participating SPNA remains which can provide the required services to the user.

\section{ANALYSIS}

\subsection{Thrashing Rate}

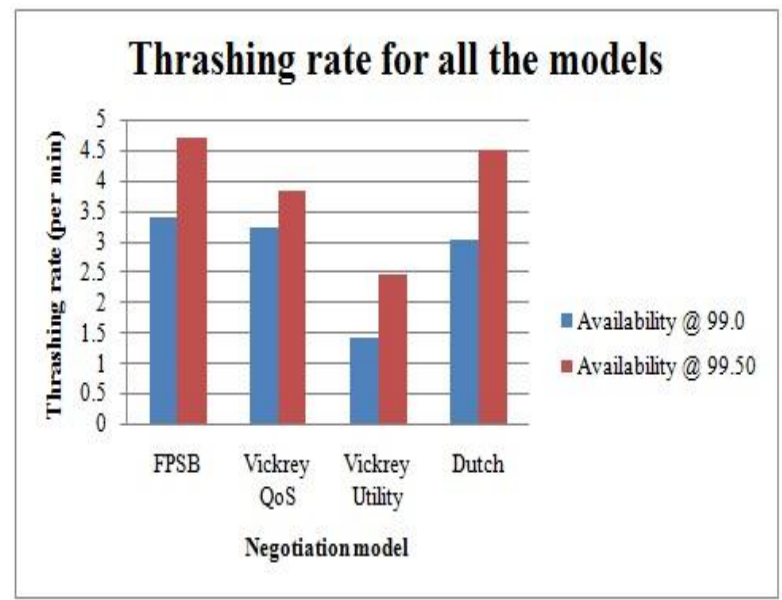

Fig 2. Thrashing rate for all the models

Figure 2 represents the comparison of thrashing at $99 \%$ and 99.5\% availability of all FPSB, Vickrey QoS, Vickrey utility and Dutch auction models. At higher levels of availability the number of SPNA who can provide services above the threshold goes on decreasing. As expected, the thrashing rate increases when availability increases. This behavior was consistent with all the auction models.

Also, the thrashing rate is least for Vickrey utility auction model. This is because of the way in which the utility function is calculated in this auction model [1].

The thrashing rate for FPSB and dutch models is similar because both models calculate the threshold based only on availability values from the respective SPNA.

The thrashing rate in Vickrey QoS is similar to dutch model but the delay in negotiation is higher for vickrey QoS than for dutch auction model. 


\subsection{Message overhead}

Figure 3 represents the comparison of number of messages exchanged by each auction model. The simulated results were averaged out for 8 negotiations.

It can be observed from the figure that Dutch auction model has the highest message overhead. This is because it undergoes multiple rounds of bidding in each negotiation.

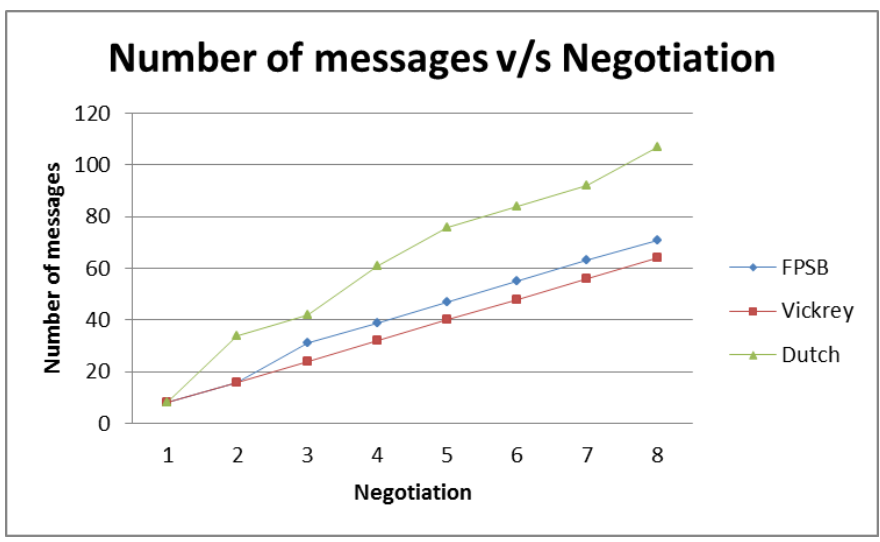

Fig 3. Message overhead for all the models

The Vickrey utility and Vickrey QoS auction models have the exact same number of messages exchanged in their respective auction processes. The difference remains in the content of the messages and the process in which the winning SPNA is calculated.

The FPSB and vickrey models have an identical number of messages exchanged in their respective auction models.

\subsection{Comparison of delay characteristics of Negotiation models}

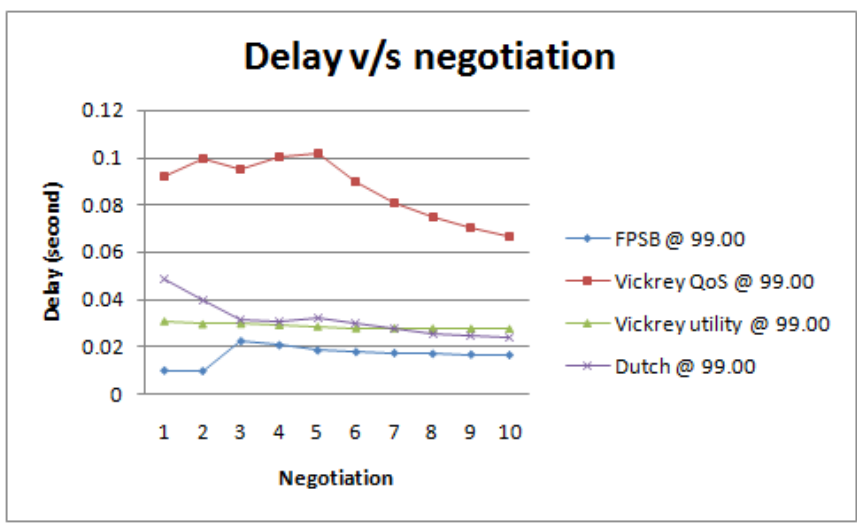

Fig 4. Delay v/s negotiations

Figure 4 indicates the delay characteristics of all negotiation models i.e. FPSB, Vickrey QoS, Vickrey utility and Dutch at $99 \%$ availability [1].

FPSB offers the least delay among the rest of the negotiation models however the number of negotiations undergone by this model over a period of 300 seconds was 24. Thus the thrashing rate is 4.8 negotiations per minute which is very high compared to all other negotiation models. The thrashing rate increases further as availability increases. Hence FPSB model is suited for systems which are sensitive to delay and stability of users to a particular service provider has low priority.

Vickrey QoS offers the highest delay among all four auction models. Also, the thrashing rate for this model was found to be near that of FPSB model. However, Vickrey QoS offered the best resource utilization for the system by calculating the winning SPNA which had the maximum available resources.

Vickrey utility offered the least thrashing rate and considerably minimal delay when compared to other negotiation models. This model is thus preferred in stable systems which require less delay. Also the message overhead of this model was similar to that of FPSB.

The Dutch model is suited for users with higher QoS requirements due to its elimination nature. As availability increased, the number of rounds required to complete the negotiation went on decreasing. However, thrashing rate went on increasing with increase in availability.

\section{CONCLUSION}

The results presented here point out the impact of message overhead and thrashing rate on the behavior of convoluted Multi Agent Systems when subjected to different negotiation models. The graphs demote that number of messages passed in First price sealed bid and Vickrey increases linearly, whereas the number of messages passed in Dutch model increase with the increase in rounds of negotiation. Also, thrashing time period increases with decrease in availability. This work concludes that there is inverse relationship between the thrashing time period and availability for the MAS architecture described in [1].

\section{FUTURE SCOPE}

By constructing formulae for message overhead and thrashing rate, the MAS architecture used above is not restricted to negotiation models discussed in this paper.

\section{REFFERENCES}

[1] Bapu Kabugade, Mohit Shroff, Nehil Shah, Srikant Panda and Dr. Nupur Giri: "Delay analysis of MAS based negotiations for cellular service providers"

[2] N. Giri, S.Bodhe, A.Ray, S.Mundle: "Multi Agent System based Service Architectures for Service Level Agreement in Cellular Networks" in press ACMCOMPUTE'09 Bangalore.

[3]Java Agent Development Framework (JADE) http://www.jade.tilab.com

[4] Distributed Operating Systems Concepts and Design by Pradeep K. Sinha (for thrashing)

[5] An Introduction to Multiagent Systems Michael Wooldridge, Department of Computer Science, University of Liverpool, UK

[6] FIPA Abstract Architecture Specification, Foundation for Intelligent Physical Agents, 2002, www.fipa.org/specs/fipa00001L 